\title{
Coded excitation of broadband terahertz using optical rectification in poled lithium niobate
}

\author{
T. Buma ${ }^{\text {a) }}$ and T. B. Norris \\ Center for Ultrafast Optical Science, University of Michigan, Ann Arbor, Michigan 48109-2099
}

(Received 30 June 2005; accepted 24 October 2005; published online 14 December 2005)

\begin{abstract}
We demonstrate coded excitation of broadband terahertz for imaging applications. The encoded transmitter uses optical rectification of femtosecond laser pulses in poled lithium niobate patterned with a 53-bit binary phase code. The terahertz wave forms are detected by electro-optic sampling in zinc telluride. A digital pulse compression filter decodes the binary wave forms, producing broadband pulses at $1.0 \mathrm{THz}$. A two-dimensional imaging experiment shows comparable performance between the encoded transmitter and a zinc telluride emitter. (C) 2005 American Institute of Physics. [DOI: 10.1063/1.2149969]
\end{abstract}

There is presently considerable interest in using terahertz $(\mathrm{THz})$ electromagnetic pulses for imaging. ${ }^{1-7} \mathrm{~A}$ common method to generate single-cycle THz pulses is optical rectification of a femtosecond laser pulse traveling through an electro-optic crystal. Maximum efficiency occurs when the optical and $\mathrm{THz}$ pulses propagate through the crystal with the same group velocity. This is satisfied with zinc telluride (ZnTe) illuminated by a Ti:Sapphire laser operating near $820 \mathrm{~nm} .{ }^{8}$ Materials for velocity matched optical rectification have been elusive for femtosecond lasers operating at other wavelengths. In velocity mismatched crystals, the rapid walkoff between the optical and THz pulses limits THz generation efficiency. The bulk of the crystal is therefore wasted, leaving only the entrance and exit crystal faces contributing to the emitted THz. We demonstrate a technique using velocity mismatched crystals to generate broadband $\mathrm{THz}$ pulses, where the entire crystal length constructively contributes to the process.

The technique employs coded excitation, where the emitted $\mathrm{THz}$ is a long encoded wave form instead of a single-cycle pulse. Coded excitation can be used with a variety of encoding wave forms. Binary phase codes are particularly attractive for systems with limited capabilities for amplitude and frequency modulation. These codes consist of the same pulse repeated at regular intervals, where the sign of the pulse is either +1 or -1 . The bandwidth of the overall code scales with the bandwidth of an individual pulse.

Binary phase codes of electromagnetic $\mathrm{THz}$ wave forms can be generated by optical rectification in poled lithium niobate (PLN). A femtosecond laser pulse traveling through a crystal domain generates a THz nonlinear polarization. The sign of the radiated $\mathrm{THz}$ field is determined by the poling direction of the domain. Group-velocity mismatch causes the optical and $\mathrm{THz}$ pulses to walk off each other as they propagate through the crystal. Poling domains separated by more than a walk-off length radiate $\mathrm{THz}$ independently from each other. This makes it possible to generate simple narrow-band $\mathrm{THz}$ by periodically alternating the poling direction of domains with equal lengths. ${ }^{9}$ More complicated wave forms are possible by engineering the poling domain structure of the crystal. ${ }^{10}$ Assigning a binary code to the crystal therefore produces a binary phase encoded $\mathrm{THz}$ wave form. The re-

\footnotetext{
${ }^{a)}$ Electronic mail: takbuma@eecs.umich.edu
}

ceived $\mathrm{THz}$ signals are stored and decoded by a digital filter, compressing the encoded wave forms to produce short pulses with high peak power. Digital pulse compression is basically an inverse filtering process, making it extremely important to use a binary code with a well-behaved spectrum (i.e., no large amplitude spikes or dips). We have chosen a 53-bit binary code shown to be an optimal invertible sequence. ${ }^{11}$

The experimental setup is illustrated in Fig. 1. The pump laser is a home-built $250 \mathrm{kHz}$ regeneratively amplified Ti:Sapphire system producing $100 \mathrm{fs}$ pulses at $800 \mathrm{~nm}$. The pump beam is acousto-optically chopped at $65 \mathrm{kHz}$ before it is focused onto the PLN crystal with a $40 \mathrm{~cm}$ focal length lens. The pump pulse energy is approximately $600 \mathrm{~nJ}$. The generated $\mathrm{THz}$ is collimated and refocused by two metallic off-axis parabolas. $\mathrm{THz}$ detection is performed with a standard electro-optic setup. ${ }^{4}$ The probe laser is first sent through a rapid scanning delay line covering a $140 \mathrm{ps}$ window at a repetition rate of $2.5 \mathrm{~Hz}$. The probe pulse energy is approximately $60 \mathrm{~nJ}$. The probe beam then passes through a GlanThompson polarizer and reflects off a pellicle to copropagate with the THz. The THz-induced birefringence inside the $\mathrm{ZnTe}$ sensor crystal modulates the polarization of

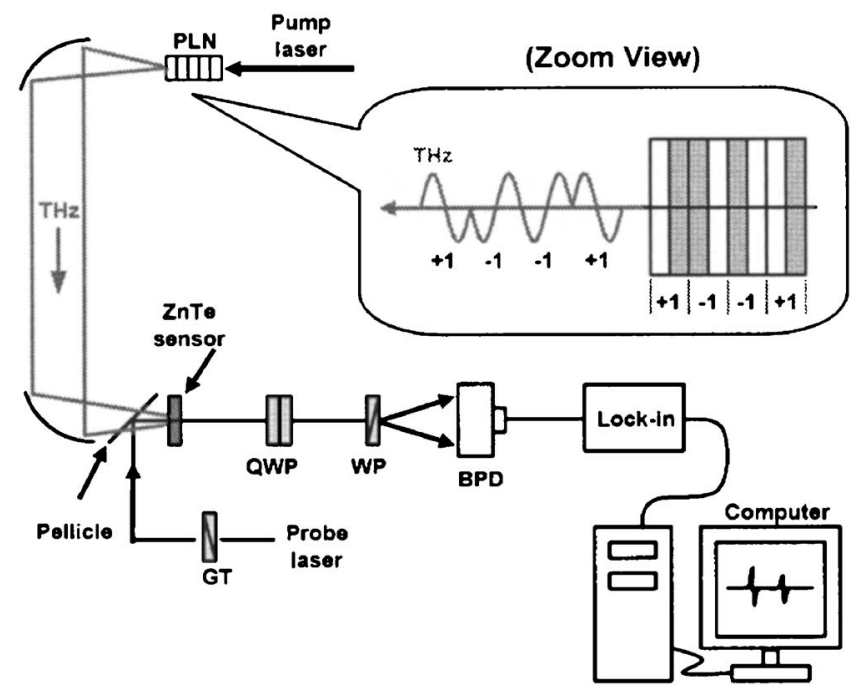

FIG. 1. Experimental setup for coded excitation of broadband terahertz. The emitter is a PLN crystal. The optical probe beam passes through a GlanThompson (GT) polarizer, a quarter-wave plate (QWP) and a Wollaston prism (WP) before illuminating a balanced photodetector (BPD). 

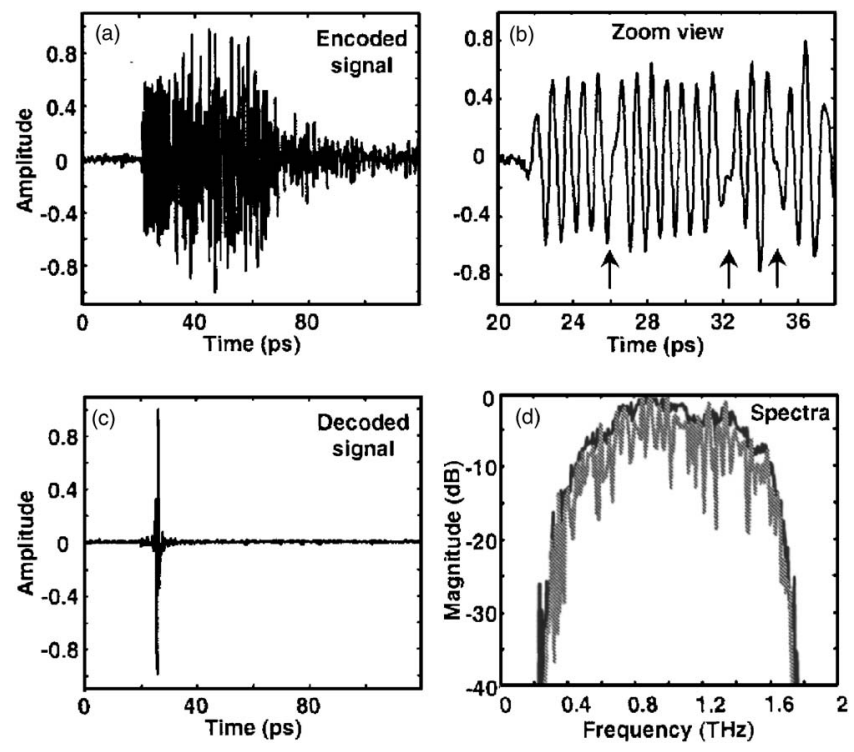

FIG. 2. Pitch-catch measurement of coded excitation. (a) Encoded wave form. The $x$ axis is time in ps and the $y$ axis is the normalized signal amplitude. (b) Zoom view of a portion of the encoded signal. The arrows indicate phase flips in the signal. (c) Digitally compressed signal. (d) Spectra of the two signals. The encoded and decoded spectra are shown by the gray and black curves, respectively. The $x$ axis is frequency in $\mathrm{THz}$, while the $y$ axis is normalized magnitude in $\mathrm{dB}$.

the probe laser. A quarter-wave plate followed by a Wollaston prism produces two optical beams for balanced detection. The balanced signal is fed through a lock-in amplifier (30 $\mu$ s time constant) and digitized by a 16-bit analog/digital board at a sampling rate of $100 \mathrm{kS} / \mathrm{s}$.

The PLN crystal, custom fabricated by Deltronic Crystal Industries, ${ }^{12}$ is $5 \mathrm{~mm}$ long and $0.5 \mathrm{~mm}$ thick. The inset of Fig. 1 depicts a portion of the crystal, where each bit consists of a pair of domains with opposite poling directions. The length of each bit is $L_{b}=94 \mu \mathrm{m}$, so each poling domain is $47 \mu \mathrm{m}$ long. The peak $\mathrm{THz}$ frequency is expected to be $f_{\text {peak }}=c /\left(n_{T}-n_{o}\right) L_{b},{ }^{9}$ where $n_{T}$ and $n_{o}$ are the THz and optical indices of refraction, respectively. Assuming $n_{T}=5.2$ and $n_{o}$ $=2.3$, we find $f_{\text {peak }}=1.1 \mathrm{THz}$. Since lithium niobate has significant $\mathrm{THz}$ absorption at room temperature, ${ }^{9}$ the PLN crystal was cooled in a liquid-helium cryostat. The exit window of the cryostat is made of high-density polyethylene, blocking the residual optical beam while transmitting most of the generated $\mathrm{THz}$.

The first experiment was a straight "pitch-catch" measurement, where no object was placed in the THz beam path. A measurement of the encoded wave form, without signal averaging, is shown in Fig. 2(a). This signal was digitally band-pass filtered between 0.3 and $1.7 \mathrm{THz}$ to suppress outof-band noise. Decoding was performed with an inverse filter in the frequency domain. ${ }^{13}$ The raw (no band-pass filtering) signal used to produce Fig. 2(a) was digitally compressed to produce Fig. 2(c). Clearly, a tight pulse is produced, along with a reduction in the noise level. The digital pulse compression is a form of signal averaging, since the individual pulses in the binary code are effectively "realigned" and added together. The spectra of the compressed and encoded signals are shown by the black and gray curves in Fig. 2(d), respectively. The ripples in the encoded signal spectrum are due to interference between the temporally separated pulses (the "bits") of the binary wave form. The digital compression

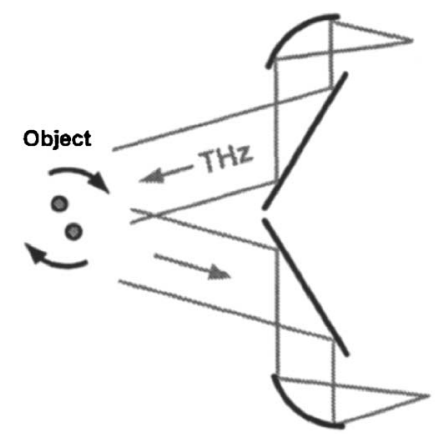

(a)

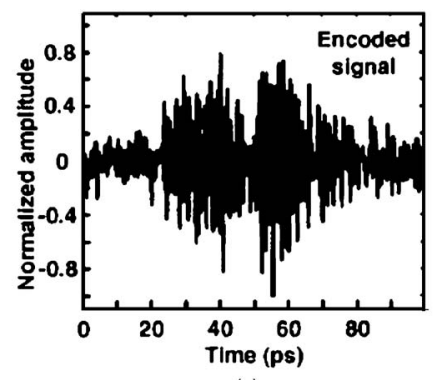

(c)

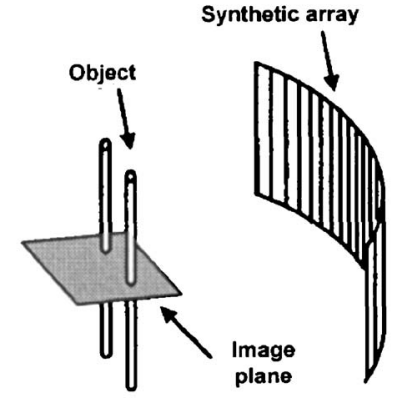

(b)

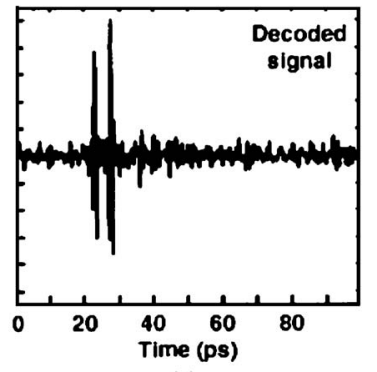

(d)
FIG. 3. (a) Geometry for two-dimensional imaging. (b) Synthetic terahertz array and the image plane. (c) An encoded signal from the data set. (d) The same signal after decoding by pulse compression filter.

filter removes most of the ripples in the encoded signal spectrum to produce a much smoother spectrum. This is in contrast to decoding with a matched filter, where spectral ripples remain after multiplication by the complex conjugate spectrum. The power spectrum of the decoded signal has a peak frequency of $0.9 \mathrm{THz}$, a mean frequency of $1.0 \mathrm{THz}$, and $a$ $-3 \mathrm{~dB}$ bandwidth of $0.85 \mathrm{THz}$.

Ideally, the decoding filter is derived directly from the encoding binary sequence, ${ }^{13}$ assuming the transmission of a uniform amplitude phase modulated wave form. There are several reasons why the signal in Fig. 2(a) deviates from this ideal behavior. Since the THz wave is not guided in the PLN, nonuniform diffraction distorts the measured wave form. The $\mathrm{ZnTe}$ sensor crystal is not an ideal detector due to $\mathrm{THz}$ absorption, velocity mismatch, and Fresnel reflections. THz absorption due to water vapor in the air introduces undesirable ringing in the detected wave form. The desired $\mathrm{THz}$ code should be 53 ps in duration. However, Fig. 2(a) shows a weaker secondary wave form starting at $75 \mathrm{ps}$ and persisting throughout the rest of the acquisition. This is due to the backward-generated $\mathrm{THz}$ inside the PLN crystal. ${ }^{9}$ A digital filter properly compressing the desired code, which is the forward-generated $\mathrm{THz}$, will transform the secondary wave form into an undesirable ringing artifact.

Applying the theoretically derived decoding filter to the distorted wave form in Fig. 2(a) produced an undesirable compressed pulse with excessively high and long-lasting sidelobes. As a result, a digital filter was derived heuristically in the following manner. First, a separate pitch-catch measurement was made with 1000 signal averages to produce a clean signal. The fast Fourier transform of this signal produces a spectrum with rapidly fluctuating phase and amplitude. The phase variations were replaced with a linear phase, while the fluctuating amplitude was smoothed out with a moving average filter. This well-behaved spectrum was then divided by the original signal spectrum, where excessive 


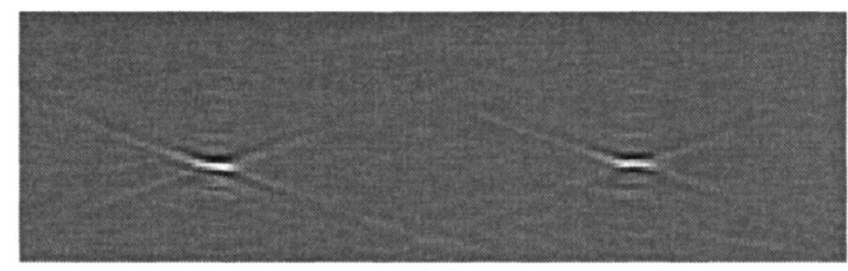

(a)

\section{$2 \mathrm{~mm}$}

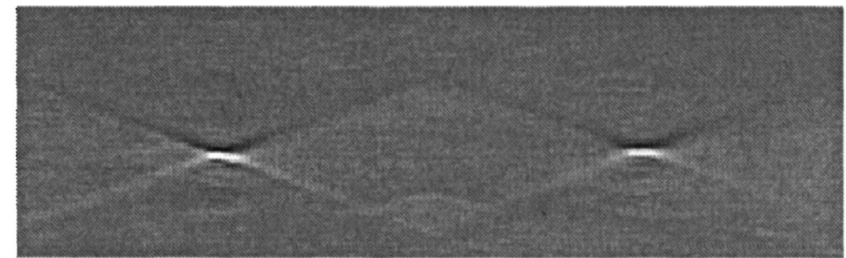

(b)

FIG. 4. (a) Reconstructed image from decoded data set using PLN emitter. (b) Reconstructed image using a $\mathrm{ZnTe}$ emitter. The scale bar represents $2 \mathrm{~mm}$ in the image plane.

noise spikes from the spectrum inversion were suppressed by bandpass filtering between 0.3 and $1.7 \mathrm{THz}$. The final result is the inverse filter for pulse compression. This filter was then applied to the signal of Fig. 2(a) to produce the compressed pulse in Fig. 2(c).

Coded excitation is a well-established technique in the gigahertz frequency range of pulse-compression radar. ${ }^{14} \mathrm{We}$ extend this technique to imaging at $\mathrm{THz}$ frequencies. The coded excitation scheme was tested with an experiment for two-dimensional (2D) time-reversal imaging. ${ }^{1-3}$ The setup is shown in Fig. 3(a), which is similar to Fig. 1. The main difference is the two metal-coated mirrors in between the off-axis parabolas. The first mirror directs the encoded terahertz onto the object, while the second mirror directs a portion of the scattered $\mathrm{THz}$ onto the second parabola for detection. The object consists of two steel drill bits spaced $5 \mathrm{~mm}$ apart. An array is synthesized by rotating the object while the transmitter and receiver remain fixed. The effective transceiver aperture, depicted in Fig. 3(b), is a curvilinear array with an $f / \#$ of 1.46 .

A wave form from the acquired data set is shown in Fig. 3(c). All signals were averaged sixteen times before storage. The average signal-to-noise ratio (SNR) of the signals is computed to be $7.5 \mathrm{~dB}$. Applying a pulse compression filter produces the wave form in Fig. 3(d), producing two clearly identifiable pulses. The average SNR of the compressed signals is computed to be $20.5 \mathrm{~dB}$, representing an improvement of $13 \mathrm{~dB}$. This same compression filter was applied to all the acquired signals before performing image reconstruction. The final linear gray-scale image is shown in Fig. 4(a), clearly showing the two objects. The field of view is 3 $\times 10 \mathrm{~mm}$. The $\mathrm{X}$ shape of the objects, often called a "bow tie" in ultrasound imaging, is characteristic of broadband pulses focused by a finite size aperture. ${ }^{15}$ For comparison purposes, a separate imaging experiment used a ZnTe emitter to transmit near single-cycle terahertz pulses. The reconstructed image is shown in Fig. 4(b) with the same linear gray scale. Both images clearly have similar SNR, indicating the encoded PLN shows comparable imaging performance to ZnTe. On the other hand, an uncoded bulk lithium niobate crystal would produce a significantly noisier image than a ZnTe emitter.

It must be emphasized that coded excitation is not intended to replace $\mathrm{ZnTe}$ for $\mathrm{THz}$ generation using optical pulses near $800 \mathrm{~nm}$. Instead, coded excitation enables broadband $\mathrm{THz}$ generation with other laser sources, such as femtosecond fiber lasers at 1 and $1.5 \mu \mathrm{m}$ wavelengths. This technique decouples the requirements for a crystal to have both velocity matching as well as high nonlinear $d_{i j k}$ coefficients.

In summary, coded excitation of broadband terahertz has been performed using optical rectification in poled lithium niobate patterned with a 53-bit binary phase code. Digital pulse compression produces broadband terahertz pulses at 1.0 THz. Coded excitation using a velocity mismatched crystal has imaged 2D objects with comparable performance to a $\mathrm{ZnTe}$ emitter. Future work will apply this method to threedimensional imaging. A PLN waveguide structure should significantly improve the fidelity of the transmitted terahertz code. Furthermore, coded excitation can be applied to a variety of nonlinear optical materials not requiring cryogenic cooling. ${ }^{16}$

${ }^{1}$ A. B. Ruffin, J. Decker, L. Sanchez-Palencia, L. Lehors, J. F. Whitaker, T. B. Norris, and J. V. Rudd, Opt. Lett. 26, 681 (2001).

${ }^{2}$ A. B. Ruffin, J. V. Rudd, J. Decker, L. Sanchez-Palencia, L. Lehors, J. F. Whitaker, and T. B. Norris, IEEE J. Quantum Electron. 38, 1110 (2002).

${ }^{3}$ T. Buma and T. B. Norris, Appl. Phys. Lett. 84, 2196 (2004).

${ }^{4}$ B. Ferguson, S. Wang, D. Gray, D. Abbot, and X. C. Zhang, Opt. Lett. 27, 1312 (2002).

${ }^{5}$ J. O'Hara and D. Grischkowsky, Opt. Lett. 27, 1070 (2002).

${ }^{6}$ T. D. Dorney, J. L. Johnson, J. V. Rudd, R. G. Baraniuk, W. W. Symes, and D. M. Mittleman, Opt. Lett. 26, 1513 (2001).

${ }^{7}$ K. McClatchey, M. T. Reiten, and R. A. Cheville, Appl. Phys. Lett. 79, 4485 (2001).

${ }^{8}$ Q. Wu and X.-C. Zhang, IEEE J. Quantum Electron. 2, 693 (1996).

${ }^{9}$ Y.-S. Lee, T. Meade, V. Perlin, H. Winful, T. B. Norris, and A. Galvanauskas, Appl. Phys. Lett. 76, 2505 (2000).

${ }^{10}$ Y.-S. Lee, N. Amer, and W. C. Hurlbut, Appl. Phys. Lett. 82, 170 (2003).

${ }^{11}$ J. Ruprecht and M. Rupf, IEEE Trans. Inf. Theory 42, 1604 (1996).

${ }^{12}$ Deltronic Crystal Industries Inc., Dover, NJ.

${ }^{13}$ Y. Wang, K. Metzger, D. N. Stephens, G. Williams, S. Brownlie, and M. O'Donnell, IEEE Trans. Ultrason. Ferroelectr. Freq. Control 50, 805 (2003).

${ }^{14}$ M. I. Skolnik, Radar Handbook, 2nd ed. (McGraw-Hill, New York, 1980).

${ }^{15}$ Z. Jiang and X.-C. Zhang, Opt. Express 5, 243 (1999).

${ }^{16}$ K. L. Vodopyanov, M. M. Fejer, D. M. Simanovskii, V. G. Kozlov, and Y. $\mathrm{S}$. Lee, Technical Digest of the Conference on Lasers and Electro-Optics (Optical Society of America, Washington, DC, 2005), paper CWM1. 